\title{
The Cost Effectiveness of Health Insurance
}

\author{
Peter Muennig, MD, MPH, Peter Franks, MD, Marthe Gold, MD, MPH
}

Background: Although studies have examined both the adverse consequences of lacking health insurance and the costs of insuring the uninsured, there are no estimates of the value of providing health insurance to those currently uninsured.

Objective: To examine the value associated with providing insurance to those currently uninsured through an incremental cost-effectiveness analysis.

Methods: People aged 25 to 64 in both the National Health Interview Survey (with 2-year mortality follow-up) and the Medical Expenditure Panel Survey were examined to estimate the contribution of sociodemographic, health, and health behavior characteristics on insured persons' quality-adjusted life years (QALYs) and healthcare costs. Parameter estimates from these regression models were used to predict QALYs and costs associated with insuring the uninsured, given their characteristics for 1996. Markov decision-analysis modeling was then employed to calculate incremental cost-effectiveness ratios.

Results: The incremental cost-effectiveness of insurance for the average 25-year-old adult (through age 64) is approximately $\$ 35,000$ per QALY gained (range $\$ 21,000$ to $\$ 48,000$ ). The incremental cost-effectiveness ratio becomes more favorable as people approach age 65 .

Conclusions: The additional health care purchased with health insurance provides gains in qualityadjusted life at costs that compare favorably to those of other programs and medical interventions society now chooses to fund.

(Am J Prev Med 2005;28(1):59-64) @ 2005 American Journal of Preventive Medicine

\section{Introduction}

I $\mathrm{n}$ the United States, the recent growth of the uninsured population, coupled with double-digit increases in insurance rates, have again galvanized discussion on the expansion of health insurance. ${ }^{1,2} \mathrm{~A}$ relevant policy concern with the addition of any new program is what benefits will be gained and at what cost. Although achievement of universal coverage may be a desirable policy goal, ${ }^{3}$ insuring the uninsured will likely raise costs further. Recent regulations set forth by the Office of Management and Budget instruct federal agencies to conduct cost-effectiveness analyses when exploring "major health and safety rules whose economic budget impact will exceed one billion dollars." Cost-effectiveness analysis permits an exploration of how new expenditures on healthcare coverage compare to other types of social investments that are purchased on behalf of the public. ${ }^{5}$

From the Department of Health Policy and Management, Mailman School of Public Health, Columbia University (Muennig), New York, New York; Center for Health Services Research in Primary Care, Department of Family and Community Medicine, University of California-Davis (Franks), Davis, California; Community Health and Social Medicine, City University of New York Medical School (Gold), New York, New York

Address correspondence and reprint requests to: Peter Muennig, MD, MPH, Mailman School of Public Health, 600 W. 168th St., 6th Fl., Columbia University, New York NY 10032. E-mail: petermuennig@ yahoo.com.
Only one randomized controlled trial of health insurance has been conducted that directly examines the impact of insurance on healthcare utilization. This study is over 20 years old, and the study groups differed only by relatively small copayment amounts. ${ }^{6}$ The limited scope of that study precludes drawing useful policy conclusions regarding the economic efficiencies associated with expanding health insurance. Observational studies that adjust for the sociodemographic and behavioral characteristics distinguishing the insured from the uninsured provide the only alternative to examining the value of expanding health insurance coverage. ${ }^{7-10}$ Two key assumptions underlie inferences made from these studies: (1) uninsured persons have reduced access to effective health care and consequently suffer adverse health effects; and (2) providing insurance increases access, thereby yielding the benefits of access to effective care.

Acknowledging the limitations of observational studies, there is abundant evidence that persons lacking health insurance delay or forgo care, and this results in worse health outcomes including lower health status, greater morbidity, and higher mortality. ${ }^{7,11}$ Evidence is more limited about the likely impact of providing health insurance to the uninsured. Some information suggests that individuals who gain insurance use health services in a manner similar to those previously insured. ${ }^{12,13}$ 
There are recent national data on the likely costs of providing insurance to the uninsured ${ }^{7,11,14}$; however, there is no information about its value relative to the benefits it may confer. Extant studies on the health effects associated with lack of insurance are dated and do not provide information in a format that can be used for a cost-effectiveness analysis. ${ }^{8-10}$ This study examines nationally representative data to examine the cost effectiveness of providing health insurance to adults aged 25 to 64 .

\section{Methods \\ Overview and Definitions}

To estimate the cost-effectiveness of insuring those currently uninsured, a three-step process was employed. First, regression analyses using nationally representative data of privately insured persons aged 25 to 65 modeled the effects of sociodemographic and clinical variables on expenditures, healthrelated quality of life (HRQL) values, and mortality. Parameter estimates from these analyses were then applied to the sociodemographic and clinical characteristics of the uninsured to predict the costs and health benefits associated with insuring the uninsured population of the United States. Finally, Markov modeling was used to estimate the costs and benefits of providing health insurance through age 65 to the average uninsured 25-year-old. A technical appendix that provides details of the data sources, regression models, and the Markov models is available at http://www.pceo.org/ insurancecea.htm.

The quality-adjusted life year (QALY) was used as an outcome measure. A year of life lived in perfect health is scored as 1 QALY. The incremental cost per QALY gained is the additional money spent on health insurance divided by the additional gains in quality-adjusted life expectancy, called the incremental cost-effectiveness ratio (ICER). The present study was conducted from the societal perspective, employed a community-derived measure of HRQL to calculate effectiveness in terms of QALYs, and used a 3\% discount rate (on future costs, life years, and QALYs) following recommendations of the Panel on Cost-Effectiveness in Health and Medicine. ${ }^{15}$ Over-the-counter drug costs, costs associated with institutionalized people, and direct nonmedical costs were not included in the analysis. ${ }^{16}$

\section{Data Sets}

The1993 National Health Interview Survey (NHIS), ${ }^{17}$ the NHIS linked to the National Death Index (NDI) through $1995^{18}$ (the most current nationally representative sources for information on the relationships between health insurance and HRQL and mortality), and the 1996 Medical Expenditure Panel Survey ${ }^{19}$ (MEPS) were respectively used to estimate HRQL scores, mortality rates, and costs after adjustment for possible confounding. The NHIS is a nationally representative sample of self-reported demographic information, illnesses, conditions, and health status of noninstitutionalized people. ${ }^{17}$ The NDI contains all deaths in the United States since 1975 and uses personal identifiers to accurately link records to survey data. ${ }^{18,20}$ Approximately $96 \%$ of respondents provided information on all outcomes of interest. In order to avoid biasing estimates toward more favorable costeffectiveness ratios, subjects who reported having no insurance due to poor health, who lost their insurance due to unemployment, and those with Medicare, Medicaid, or other types of government insurance were excluded. This yielded 24,578 subjects with 140 deaths through 1996.

Health-related quality-of-life scores were derived from the Health and Activities Limitation Index (HALex). The HALex is comprised of two health domains, self-rated health and role limitations, that were obtained from the NHIS and combined using correspondence analysis and multiattribute utility scaling. ${ }^{21}$ The values from these analyses are then linked to the Health Utility Index. The HALex exhibits reasonable validity (see also the technical appendix), but is less sensitive to the full spectrum of morbidity than other measures..$^{22}$ Details of these methods have been published elsewhere. ${ }^{21}$

\section{Regression Analyses}

Regression models were developed using SUDAAN 8.0.1 (Research Triangle Institute, Research Triangle Park NC, 2001) and STATA 8.2 (Stata Corp, College Station TX, 2003), adjusting for the complex sample designs used in both NHIS and MEPS. In these models, HRQL scores, survival, or total medical expenditures were entered as dependent variables. Age, race/ethnicity, gender, education, family income (NHIS), or percent of federal poverty level (MEPS), family size, marital status, employment status, behavioral risk factors (smoking status and seatbelt use in NHIS), number of conditions reported (NHIS) or self-rated health (MEPS), and area of residence (region of country, and urban or rural location) were entered as independent variables. In the mortality and HRQL models (NHIS/NDI), people who were uninsured because of illness or loss of work were excluded, and insurance status was defined as that reported at the time of interview. In the expenditure models (MEPS), only people whose insurance status did not change throughout the year were included.

The effects of the sociodemographic and health variables on HRQL scores were estimated using linear regression. Expenditures were analyzed using generalized linear regression models with a gamma distribution and a log link function (to account for the skewed distribution of expenditures). The resulting parameter estimates were used to calculate predicted HRQL scores and expenditures for the uninsured using their sociodemographic and health characteristics.

Proportional hazard models were used to estimate the risk of death due to lacking health insurance. Hazard ratio estimates were unstable when the behavioral risk factors were included since including them reduced the sample size. However, behavioral risk factors exerted little effect on differences in mortality by insurance status. The larger sample was therefore used, and the hazard ratios were reduced by the $10 \%$ effect size of the behavioral risk factors (see technical appendix).

The predicted expenditures for the insured were adjusted to include administrative costs for health insurance plans. In order to appropriately link expenditure data to outcome data, all costs were deflated to constant 1994 dollars using the medical portion of the consumer price index. 
Table 1. Principal assumptions of analysis

1. Since it was not possible to estimate the extent to which health insurance produces long-term benefits, it was assumed that subjects would revert to average mortality rates after age 65 .

2. Although insurance status changes from year to year, no information on which uninsured subjects were previously insured or vice versa were available. It was therefore assumed that the insurance status of subjects during the year for which data were collected was static.

3. There are few data on administrative costs. It was assumed that costs associated with provider and hospital administration would be included in the overall expenditures by insurance companies and that employer administrative costs would be negligible.

4. All relevant covariates were included in the models, so that the estimates of the effects of insurance status reflect causal relationships. It is possible that association between insurance status and unmeasured variables bias the estimates of the effects of insurance on costs, mortality, and HRQL.

5. Expenditures included in the MEPS survey reflect all relevant costs. The costs reported in the MEPS do not include the institutionalized population, over-the-counter medications, or alternative care. Also excluded are transportation costs.

HRQL, health-related quality of life; MEPS, Medical Expenditure Panel Survey.

\section{Decision Analysis Models}

Markov models were built using DATA Professional, version 4.0 (TreeAge Software, Williamstown MA). The models evaluated two possible events: providing private health insurance to the uninsured or having them remain uninsured. For each health state, subjects were exposed to an annual, age-specific risk of death, with survivors gaining 1 HRQL-adjusted year and medical costs. The values for these year-to-year changes in health by insurance status were obtained from the regression analyses. Thus, the average cost of medical care for a 25-year-old with insurance is tabulated, and the model is advanced 1 year. Age-specific mortality rates are used to determine the proportion of subjects dying, and survivors are assigned discounted QALYs and costs for the next year. This process is repeated until subjects reach age 65 (whereupon they are eligible for Medicare). The same process is applied to the uninsured cohort, and incremental values are calculated. Assumptions are listed in Table 1.

The variables used in the analyses were subjected to a Monte Carlo simulation and one-way sensitivity analyses. In a one-way analysis, all variables are held constant but one. In a Monte Carlo simulation, values for all variables are randomly sampled from a statistical distribution. ${ }^{23}$ The Monte Carlo simulation used a triangular distribution. In this distribution, the base case estimate is entered as the most likely value, and values between the high and low value are linearly interpreted.

\section{Analytic Validations}

In MEPS, expenditures among the insured often reflect prenegotiated discounted charges. ${ }^{19}$ Therefore, the uninsured may pay more for similar services. Conversely, MEPS
Table 2. Observed and predicted expenditures and health outcomes

\begin{tabular}{lllll}
\hline & $\begin{array}{c}\text { Age } \\
\text { group }\end{array}$ & Insured & Uninsured & Predicted $^{\mathbf{a}}$ \\
\hline Expenditures & $25-34$ & $\$ 1169$ & $\$ 326$ & $\$ 1193$ \\
& $35-44$ & $\$ 1543$ & $\$ 519$ & $\$ 1613$ \\
& $45-54$ & $\$ 1935$ & $\$ 742$ & $\$ 2219$ \\
& $55-64$ & $\$ 2820$ & $\$ 2280$ & $\$ 3385$ \\
HRQL score & $25-34$ & 0.92 & 0.88 & 0.88 \\
& $35-44$ & 0.90 & 0.84 & 0.86 \\
& $45-54$ & 0.87 & 0.79 & 0.83 \\
Mortality & $55-64$ & 0.84 & 0.73 & 0.78 \\
& $25-34$ & 0.001 & 0.002 & 0.001 \\
& $35-44$ & 0.002 & 0.003 & 0.002 \\
& $45-54$ & 0.006 & 0.009 & 0.006 \\
& $55-64$ & 0.012 & 0.020 & 0.013 \\
\hline
\end{tabular}

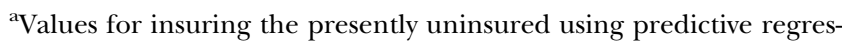
sion models.

${ }^{\mathrm{b}}$ Proportion dying within 24 months.

HRQL, health-related quality of life.

assigns zero dollars to unpaid visits. For these reasons, the actual cost of the medical goods or services consumed by the uninsured may be higher or lower than the expenditures reported in MEPS. To explore this further, per medical event expenditures were compared between those with and without private insurance for 25 service categories (e.g., hospitalizations), adjusting for sociodemographic covariates and health status, to estimate the extent and direction of any resulting bias.

The decision analysis models were validated by comparing outputs with abridged health-adjusted life tables. ${ }^{24}$ Markov modeling allows for a more accurate estimation of year-toyear effects by accounting for the effect of mortality on cost and effectiveness and simplifies sensitivity analyses. Abridged life tables allow for clear and simple estimations of changes in life expectancy when different interventions are applied, and may thus serve to validate Markov models.

\section{Results}

The observed and predicted expenditures, HRQL, and mortality for the insured, the uninsured, and the predicted values for the uninsured with insurance are shown in Table 2. For the uninsured, actual expenditures are lower, quality of life is lower, and mortality experience higher than the insured. The predicted expenditures are higher, the quality of life lower, and mortality higher for the uninsured provided insurance than for the currently insured. These findings reflect the lower socioeconomic status and greater morbidity of the currently uninsured compared with the insured.

Were the average uninsured 25-year-old to have private health insurance, discounted societal costs would increase by $\$ 30,000$ per person over the 45 -year interval to age 65 . The average 25 -year-old would gain 0.9 QALY over this interval, resulting in an ICER of $\$ 35,000 /$ QALY gained (Table 3, values rounded). The average uninsured 25-year-old would also gain 0.6 years 
Table 3. Results of cost-effectiveness analyses at a $3 \%$ discount rate

\begin{tabular}{|c|c|c|c|c|c|c|}
\hline & Strategy & Cost (US\$) & $\begin{array}{l}\text { Incremental } \\
\text { cost }\end{array}$ & Effectiveness $^{\mathbf{a}}$ & $\begin{array}{l}\text { Incremental } \\
\text { effectiveness }^{\text {a }}\end{array}$ & $\begin{array}{l}\text { Incremental } \\
\text { cost-effectiveness }^{\text {b }}\end{array}$ \\
\hline \multirow[t]{2}{*}{ Cost per QALY gained } & No insurance & $\$ 17,000$ & - & 21.7 QALYs & - & - \\
\hline & Insurance & $\$ 47,000$ & $\$ 30,000$ & 22.5 QALYs & 0.9 QALYs & $\$ 35,000$ \\
\hline Cost per LY gained & Insurance & $\$ 47,000$ & $\$ 30,000$ & 27.1 LYs & $0.6 \mathrm{LYs}$ & $\$ 51,000$ \\
\hline
\end{tabular}

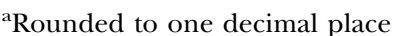

${ }^{\mathrm{b}}$ Rounded to nearest thousand.

LY, life year; QALY, quality-adjusted life year.

of life (i.e., unadjusted for quality), resulting in an ICER of approximately $\$ 51,000 /$ life year gained.

\section{Sensitivity Analyses}

Table 4 lists the effects of relevant one-way sensitivity analyses on ICERs. Over a plausible range of high and low values for model inputs, the estimated ICER of providing insurance to the uninsured varied by as much as $\$ 27,000 /$ QALY gained.

Discount rates are used to determine the value of future goods in present-day terms. The discount rate used caused the ICER to vary between $\$ 22,000 /$ QALY gained when no discounting was applied to $\$ 45,000$ / QALY gained when a 5\% rate was applied. In the Monte Carlo analyses, the ICER of insurance relative to no insurance ranged from $\$ 21,000$ to $\$ 48,000$ among people aged 25 to 65 .

The incremental cost-effectiveness ratio improves with age (Figure 1). Thus, if the uninsured were to receive private insurance beginning at age 50 , the incremental cost-effectiveness of providing private health insurance would be $\$ 18,000$ per QALY gained.

The effects of medical inflation between 1994 and 2001 on the ICER were examined under the conservative assumption of no interval improvement in the effectiveness of care. The resulting ICER would increase to $\$ 39,000 /$ QALY.

\section{Validation of Cost Estimates and Decision Analysis Models}

The per event expenditures tended to be lower for the uninsured group. In some instances, the effects were statistically significant; for example, after adjustment for sociodemographic factors and self-rated health, expenditures per outpatient visit were $\$ 30$ lower among the uninsured. Thus, for the uninsured group, unreimbursed care either outweighs uninsured persons' inability to negotiate lower rates for similar goods and services or the uninsured receive fewer services.

The Markov models predicted incremental cost, effectiveness, and cost-effectiveness values that were similar to the spreadsheet models.

\section{Discussion}

Were insurance provided to uninsured 25- to 64-yearolds, the models predict that their life expectancy would increase by about 7 months, and that their quality-adjusted life expectancy would increase by about 0.9 QALY. These gains in health and life would be comparable in cost to programs or medical interventions that are thought of as good buys. ${ }^{25}$ Moreover, such expenditures would be considerably lower than investments in many other well-accepted social programs (e.g., airline and automobile safety) in terms of cost per life year gained. ${ }^{26}$

The cost-effectiveness of health insurance increases with age. This occurs because increases in the absolute benefits associated with health insurance with age outstrip increases in health expenditures. Thus, if incremental reforms were to be implemented, such as lowering the eligibility age for Medicare, providing insurance to older persons would be more cost-effective than extending insurance to age 25 (Figure 1).

The data, extending through 1996, are somewhat dated and the extent to which gains in effectiveness offset increases in the costs of medical care in the last 8 years is unknown. The sensitivity analysis focused solely on the effects of medical inflation through 2001, and holding effectiveness at the same level as 1996, still produced favorable cost-effectiveness ratios. Increases in the benefits of health insurance have been likely over time, as medical care has presumably become more effective (also see Table 10, technical appendix). Earlier studies examining the impact of insurance status on mortality found that lacking health insurance was associated with a hazard ratio of about $1.25 .^{7-9}$ Using similar methods but data through 1996, this study found that the protective effect of insurance on mortality may have increased over the intervening 9- to 10 -year period by an additional $25 \%$.

While there may be other missing covariates, these analyses are susceptible to at least two main contravening biases. First, people with health insurance may be healthier and more health oriented, and it is those factors, rather than insurance, that are associated with improved outcomes. Although the reverse causality problem-that is, the uninsured are uninsured because 
Table 4. Variables included in the decision analysis model, their assigned high, low, and baseline values, and the effect on ICER of insurance relative to no insurance per QALY

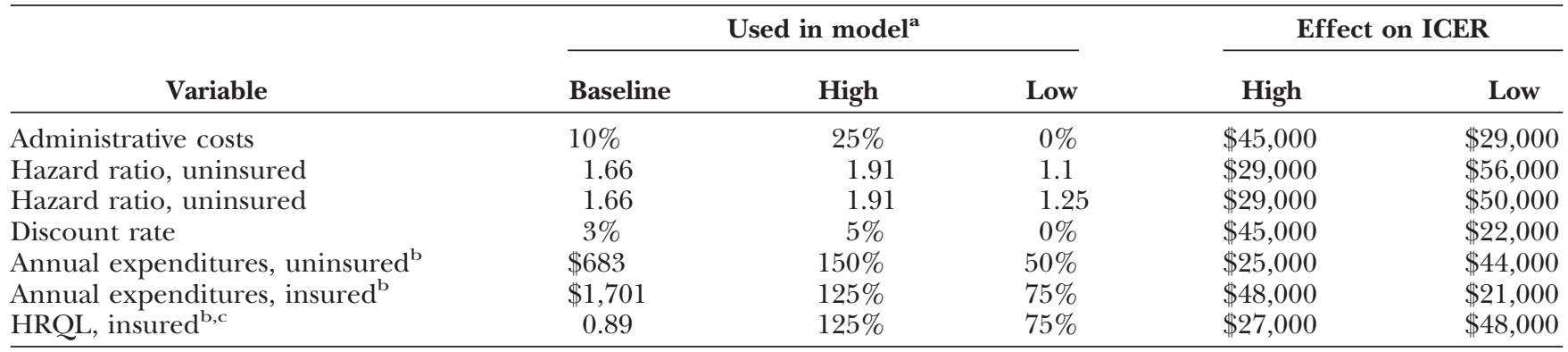

${ }^{a}$ All values represent the percentage change in the baseline value except for the hazard ratio, which was varied from previously published estimates $^{8,9}$ to the high value observed in logistic regression analysis (see technical appendix).

${ }^{b}$ Average values are presented here to conserve space. Age-specific values in 10-year intervals for the 25 to 65 cohort were used in the model. 'The range of HRQL scores was calculated by obtaining the average percentage difference in scores between the insured and uninsured cohort rather than the absolute difference.

HRQL, health-related quality of life; ICER, incremental cost-effectiveness ratio; QALY, quality-adjusted life year.

they are sicker (rather than sicker because they are uninsured) -is often cited, the evidence suggests that most people get or lose insurance as a result of their work, not of their health (also see Table 11, technical appendix). ${ }^{27}$ Second, and conversely, people choosing to have health insurance may do so because they perceive themselves to be at higher health risk than is captured in the covariates used. To control for health orientation, two behavioral risk factors, seatbelt use and smoking status, were included as covariates. However, risky health behavior can be quite nuanced. For example, there is evidence that persons with greater medical skepticism are less likely to have health insurance, use less care, and have higher mortality. ${ }^{28,29}$ While, people with lower utilization may also have lower expenditures, it is possible that the adjusted hazard ratios did not fully capture these effects.

This trade-off between health orientation and health risk highlights some of the limitations of the analysis. As with any cost-effectiveness analysis or epidemiologic

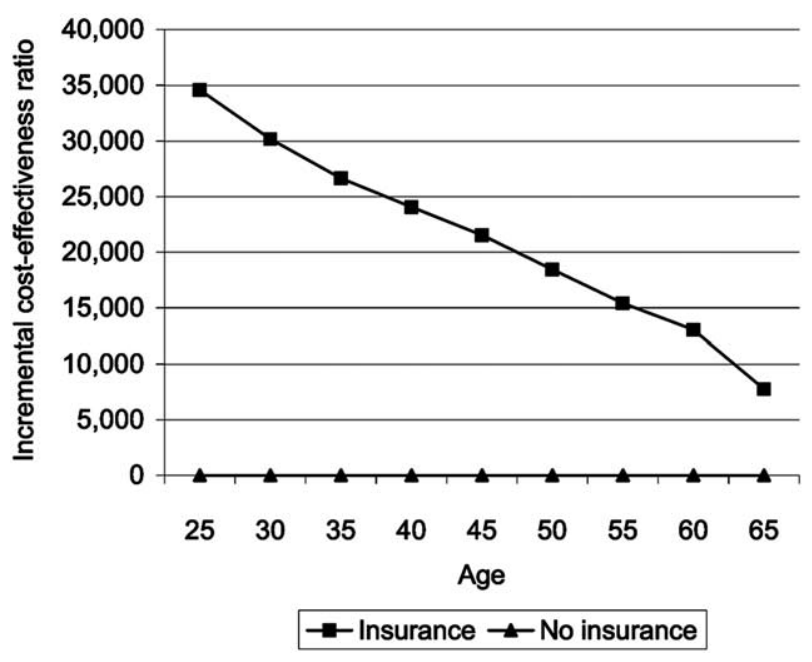

Figure 1. Changes in the incremental cost-effectiveness of insuring the uninsured cohorts at different ages. study based on observational data, the study likely contains a number of other sources of nonrandom error (e.g., measuring income rather than total wealth). Recognizing that observational data are the only available means of evaluating whether limited dollars should be allocated to health or to other public programs, these biases were balanced in a way that would be meaningful to policymakers. Known sources of bias were therefore weighed against the insurance arm of the model. Thus, if insurance were found to be within an acceptable range of incremental values, policymakers could be reasonably sure that it is costeffective. For instance, human capital losses, recently estimated in an Institute of Medicine report ${ }^{30}$ at $\$ 35$ billion to $\$ 130$ billion, were not included.

The analyses are based on an assumption of expanded coverage with the current mix of private plans that incorporate various amounts of capitation, copayments and deductibles. This is not to suggest, however, that this mix of private plans is the most efficient means of expanding health insurance. It is likely that comparable outcomes could be achieved at lower cost using alternative models of healthcare delivery. Health insurance proposals range from incremental approaches $^{31}$ to universal single payer models. ${ }^{32}$ Both the U.S. General Accounting Office and the Congressional Budget Office have concluded that universal plans may be more efficient than the current system. ${ }^{33,34}$ These data and methods can be used to evaluate such models. Guidelines for estimating the costs of incremental reform policies have been published elsewhere. ${ }^{35}$

The continued rise in the percentage of uninsured persons in the United States, and the contentious Medicare pharmaceutical benefit recently signed into law has re-ignited discussion about who to cover and what services they should receive. This study builds on a growing body of research, published both in the medical literature and by the Congressional Budget Office, that examines the costs and health effects of 
While a growing body of evidence suggests that health insurance is effective at reducing morbidity and mortality, it is not known whether the additional medical care it buys is well spent.

This study finds that this additional care costs approximately $\$ 35,000$ per quality-adjusted life year gained, which compares favorably to other programs and medical interventions that we now choose to fund.

lacking insurance. This study found that the gain in life expectancy and reduction in morbidity with health insurance were modest and within the general range of values seen in previous studies. ${ }^{8-10}$ However, these improvements come at a reasonable cost when compared to other investments that we as a society choose to fund. ${ }^{25,26}$ This suggests that it is feasible that health insurance can be expanded at a good value.

We thank Willard Manning for his guidance in suggesting approaches to the modeling of costs.

No financial conflict of interest was reported by the authors of this paper.

\section{References}

1. Strunk BC, Ginsburg PB. Tracking health care costs: trends stabilize but remain high in 2002. Health Aff (Millwood) 2003 Jan-Jun; (suppl web exclusives):266-74.

2. Mills R. Health insurance coverage 2001. Washington DC: U.S. Bureau of the Census, 2002 (Publication P60-220).

3. Institute of Medicine. Insuring America's health: principles and recommendations. Washington DC: National Academies Press, 2004.

4. Graham J. Draft 2003 report to Congress on the costs and benefits of federal regulations. Washington DC: Office of Management and Budget, 2003.

5. Weinstein MC, Stason WB. Foundations of cost-effectiveness analysis for health and medical practices. N Engl J Med 1977; 296:716-21.

6. Brook RH, Ware JE Jr, Rogers WH, et al. Does free care improve adults' health? Results from a randomized controlled trial. N Engl J Med 1983; 309:1426-34.

7. Institute of Medicine. Care without coverage: too little, too late. Washington DC: National Academies Press, 2002.

8. Sorlie PD, Johnson NJ, Backlund E, Bradham DD. Mortality in the uninsured compared with that in persons with public and private health insurance. Arch Intern Med 1994;154:2409-16.

9. Franks P, Clancy CM, Gold MR. Health insurance and mortality. Evidence from a national cohort. JAMA 1993;270:737-41.

10. Baker DW, Sudano JJ, Albert JM, Borawski EA, Dor A. Lack of health insurance and decline in overall health in late middle age. $\mathrm{N}$ Engl $\mathrm{J}$ Med 2001;345:1106-12.

11. Hadley J. Sicker and poorer-the consequences of being uninsured: a review of the research on the relationship between health insurance, medical care use, health, work, and income. Med Care Res Rev 2003;60: 3S-75S, 76S-112S.

12. Rodewald LE, Szilagyi PG, Holl J, Shone LR, Zwanziger J, Raubertas RF. Health insurance for low-income working families. Effect on the provision of immunizations to preschool-age children. Arch Pediatr Adolesc Med 1997;151:798-803.

13. McWilliams JM, Zaslavsky AM, Meara E, Ayanian JZ. Impact of Medicare coverage on basic clinical services for previously uninsured adults. JAMA 2003;290:757-64.

14. Hadley J, Holahan J. How much medical care do the uninsured use, and who pays for it? Health Aff (Millwood) 2003 Jan-Jun; (suppl web exclusives):66-81.

15. Gold M, Siegel J, Russell L, Weinstein M. Cost-effectiveness in health and medicine. New York: Oxford University Press, 1996.

16. Selden TM, Levit KR, Cohen JW, et al. Reconciling medical expenditure estimates from the MEPS and the NHA, 1996. Health Care Finance Rev 2001;23:161-78.

17. National Center for Health Statistics. National Health Interview Survey, 1993. Atlanta GA: Centers for Disease Control and Prevention, National Center for Health Statistics, 1993.

18. National Center for Health Statistics. National Death Index, 1995. Atlanta GA: Centers for Disease Control and Prevention, National Center for Health Statistics, 1995.

19. Agency for Healthcare Research and Quality. Medical Expenditure Panel Survey, 1996. Rockville MD: U.S. Department of Health and Human Services, Agency for Healthcare Research and Quality, 1996.

20. Williams BC, Demitrack LB, Fries BE. The accuracy of the National Death Index when personal identifiers other than Social Security number are used. Am J Public Health 1992;82:1145-7.

21. Erickson P, Wilson R, Shannon I. Years of healthy life. Atlanta GA: Centers for Disease Control and Prevention, 1995.

22. Gold MR, Franks P, McCoy KI, Fryback DG. Toward consistency in cost-utility analyses: using national measures to create condition-specific values. Med Care 1998;36:778-92.

23. Halpern EF, Weinstein MC, Hunink MG, Gazelle GS. Representing both first- and second-order uncertainties by Monte Carlo simulation for groups of patients. Med Decision Making 2000;20:314-22.

24. Muennig PA, Gold MR. Using the years-of-healthy-life measure to calculate QALYs. Am J Prev Med 2001;20:35-9.

25. Harvard Center for Risk Analysis. The CEA Registry. Cambridge MA: Harvard University, Harvard Center for Risk Analysis, 2004.

26. Tengs TO, Adams ME, Pliskin JS, et al. Five hundred life-saving interventions and their cost-effectiveness. Risk Analysis 1995;15:369-90.

27. Blumberg L, Nichols L. Why are so many Americans uninsured? In: McLaughlin CG, ed. Health policy and the uninsured. Washington DC: Urban Institute Press, 2004:1-365.

28. Fiscella K, Franks P, Clancy CM, Doescher MP, Banthin JS. Does skepticism towards medical care predict mortality? Med Care 1999;37:409-14.

29. Fiscella K, Franks P, Clancy CM. Skepticism toward medical care and health care utilization. Med Care 1998;36:180-9.

30. Institute of Medicine. Hidden costs, value lost: uninsurance in America Washington DC: National Acadamies Press, 2003.

31. Etheredge L. A flexible benefits tax credit for health insurance and more. Health Aff (Millwood) 2002; (suppl web exclusives):1-10.

32. Grumbach K, Bodenheimer T, Himmelstein DU, Woolhandler S. Liberal benefits, conservative spending. The Physicians for a National Health Program proposal. JAMA 1991;265:2549-54.

33. Government Accounting Office. Canadian health insurance: lessons for the United States. Washington DC: Government Accounting Office, 1991 (GAO/HRD-91-90).

34. Congressional Budget Office. Single-payer and all-payer health insurance systems using Medicare's payment rates. Washington DC: Congressional Budget Office, 1993.

35. Glied S, Remler DK, Zivin JG. Inside the sausage factory: improving estimates of the effects of health insurance expansion proposals. Milbank $Q$ 2002;80:603-35, iii. 